\section{SOBRE LAS REVISTAS MÉDICAS PERUANAS}

\section{[ABOUT PERUVIAN MEDICAL JOURNALS]}

\author{
Charles Huamaní ${ }^{1}$
}

Sr. Editor. Con interés leí el artículo de Pamo-Reyna, sobre las "Características de los trabajos publicados sobre las propiedades de las plantas en revistas médicas peruanas" (1), y aun cuando la metodología empleada no precisa los criterios para elegir las revistas, entre otras cosas, se puede considerar que la selección de publicaciones fue por conveniencia y, por tanto, el trabajo tendría como limitación describir sólo los trabajos publicados en revistas médicas peruanas, sin considerar, como bien se aclara, la probabilidad de que muchos trabajos estén siendo publicados en revistas extranjeras o en otras revistas peruanas no incluidas en su listado. Al margen de ello, no se puede discutir el gran aporte que brinda.

Sin embargo, llama la atención que en una réplica posterior, sugiere que la inclusión de las revistas se basó en el conocimiento pasivo de estas, pues declara que no incluyó la revista CIMEL porque ...Nunca ha recibido un número de la revista ${ }^{(2)}$, además de suponer otras consideraciones que, así como la primera, no son justificadas, en especial porque en la referencia de la réplica incluye una donde se le comunica la presencia de la revista CIMEL dentro de la literatura biomédica nacional ${ }^{(3)}$

Al igual que el Dr. Pamo, he venido desarrollando investigación bibliométrica, y reconozco que sus publicaciones son de las pocas que abordan el estudio de la literatura científica biomédica peruana ${ }^{(4)}$. Por ello y como parte de mi tesis Análisis de los artículos científicos en revistas médicas peruanas, 2005 - 2008, desarrollé

Tabla 1. Revistas médicas peruanas con mayores indizaciones: registro de indizaciones en bases de datos, directorios y repositorios, periodicidad y año de inicio de publicación.

\begin{tabular}{|c|c|c|c|c|}
\hline Revista & Institución & Periodicidad & Inicio & $\begin{array}{c}\text { Indizaciones / Directorios / } \\
\text { Repositorios }\end{array}$ \\
\hline $\begin{array}{l}\text { R. de Gastroenterología } \\
\text { del Perú }\end{array}$ & S. Gastroenterología del Perú & Trimestral & 1981 & $\begin{array}{l}\text { MedLine; SCOPUS; SciELO; } \\
\text { LILACS; HINARI; FMJ; SB; LX }\end{array}$ \\
\hline $\begin{array}{l}\text { R. Peruana de Medicina } \\
\text { Experimental y Salud } \\
\text { Pública }\end{array}$ & Instituto Nacional de Salud & Trimestral & 1942 & $\begin{array}{l}\text { SciELO; Redalyc; LILACS; HINARI; } \\
\text { OARE, AGORA, DOAJ, MedicLatina; } \\
\text { IM; SB; LX }\end{array}$ \\
\hline $\begin{array}{l}\text { Anales de la Facultad de } \\
\text { Medicina }\end{array}$ & $\begin{array}{l}\text { U. Nacional Mayor de San } \\
\text { Marcos. F. Medicina }\end{array}$ & Trimestral & 1918 & $\begin{array}{l}\text { Redalyc; LILACS; HINARI; DOAJ; } \\
\text { FMJ; SB; PRP; LX }\end{array}$ \\
\hline R. Médica Herediana & $\begin{array}{l}\text { U. Peruana Cayetano Heredia. } \\
\text { F. Medicina }\end{array}$ & Trimestral & 1990 & $\begin{array}{l}\text { SciELO; LILACS; HINARI; } \\
\text { MedicLatina; LX }\end{array}$ \\
\hline Acta Médica Peruana & Colegio Médico del Perú & Trimestral & 1972 & $\begin{array}{l}\text { SciELO; LILACS; HINARI; DOAJ; } \\
\text { SB; LX }\end{array}$ \\
\hline R. Peruana de Pediatría & S. Peruana de Pediatría & Trimestral & 1927 & LILACS; SB; PRP; LX \\
\hline R Dermatología Peruana & S. Peruana de Dermatología & Trimestral & 1996 & $\begin{array}{l}\text { LILACS; HINARI; DOAJ; SB; PRP; } \\
\text { LX }\end{array}$ \\
\hline $\begin{array}{l}\text { R. de la Sociedad Peruana } \\
\text { de Medicina Interna }\end{array}$ & S. Peruana de Medicina Interna & Trimestral & 1998 & LILACS; HINARI; SB; LX \\
\hline Diagnóstico & $\begin{array}{l}\text { Fundación Instituto Hipólito } \\
\text { Unanue }\end{array}$ & Trimestral & 1977 & LILACS; LX \\
\hline $\begin{array}{l}\text { Ciencia e Investigación } \\
\text { Médica Estudiantil } \\
\text { Latinoamericana (CIMEL) }\end{array}$ & $\begin{array}{l}\text { S. Científica de San Fernando, } \\
\text { Federación Latinoamericana } \\
\text { de Sociedades Científicas de } \\
\text { Estudiantes de Medicina }\end{array}$ & Semestral & 1996 & Redalyc; DOAJ; IM; SB; PRP; LX \\
\hline
\end{tabular}

$\mathrm{R}=$ Revista, $\mathrm{U}=$ Universidad, $\mathrm{S}$ = Sociedad, F = Facultad; Dir/Repos = Directorios y repositorios; SB = SISBIB-UNMSM, IM = IMBIOMED, LX: Latindex Catálogo; PRP: Portal Revistas Peruanas, de CONCYTEC. Se consideran las revistas científicas con al menos una edición en 2008 e indizadas en las bases de datos o catálogos: Medline, Scopus, SciELO-Perú, Redalyc, LILACS, HINARI, DOAJ, FMJ, MedicLatina o Latindex.

Modificado de: Huamaní C, Pacheco-Romero J. Visibilidad y Producción de las Revistas Biomédicas Peruanas. Rev Gastroenterol Perú. 2009; 29(2):132-9. Revisión realizada en enero 2009.

Médico cirujano, Sociedad Científica de San Fernando, Facultad de Medicina, Universidad Nacional Mayor de San Marcos. Lima, Perú. 
el artículo Visibilidad y producción de las revistas biomédicas peruanas ${ }^{(5)}$, que me sirvió de referencia para poder seleccionar las revistas con más trayectoria, periodicidad y visibilidad (Tabla 1); además de actualizar la tesis desarrollada inicialmente por el Dr. Pamo ${ }^{(6)}$.

La revista CIMEL, es la única revista médica editada por estudiantes, que se mantiene vigente en el Perú, habiendo logrado conseguir indizaciones diversas, lo cual demuestra de forma indirecta, el prestigio y rigurosidad con la que se desarrolla ${ }^{(5)}$. Esta revista es editada por la Sociedad Científica de San Fernando, asociación estudiantil sanfernandina preocupada por el desarrollo de la investigación médica nacional. ¿Es acaso menos literatura por haber sido editada por estudiantes? Ejemplos de trabajos estudiantiles reconocidos hay varios, y sobre revistas recordemos la Crónica Médica editada al igual por estudiantes sanfernandinos en $1884{ }^{(6)}$, componentes de la Unión Fernandina.

Considero que se debe tener cuidado al hacer apreciaciones sobre entidades o instituciones, sobre todo al basarse en indicadores generales que no evalúan la calidad de una revista científica, como sí lo pueden ser las indizaciones; de igual manera se debe ser abierto a nuevas evaluaciones y diseños, que a veces no son tan nuevos.

Espero que para futuros estudios sobre revistas biomédicas peruanas se incluya, si los criterios de inclusión así lo permiten, a la revista CIMEL, y se considere a los trabajos con participación estudiantil como trabajos con limitaciones y potenciales, como cualquier otro trabajo.

\section{Conflicto de Interés}

$\mathrm{CH}$ fue miembro del comité editorial de la revista

CIMEL de 2005 a 2007.

\section{REFERENCIAS BIBLIOGRÁFICAS}

1. Pamo-Reyna OG. Características de los trabajos publicados sobre las propiedades de las plantas en revistas médicas peruanas. Rev Peru Med Exp Salud Publica. 2009; 26(3): 314-23.

2. Pamo O. Estado actual de las publicaciones científicas. Rev Med Hered. 2005; 16(3): 228-30.

3. Pamo-Reyna OG. Publicaciones estudiantiles sobre propiedades medicinales de las plantas en Perú. Réplica. Rev Peru Med Exp Salud Publica. 2009; 26(4): 586-87.

4. Pamo O. Estado actual de las publicaciones periódicas científicas médicas del Perú. Rev Med Hered. 2005; 16(1): 65-73.

5. Huamaní C, Pacheco-Romero J. Visibilidad y producción de las revistas biomédicas peruanas. Rev Gastroenterol Perú. 2009; 29(2): 132-9.
6. Pamo Reyna OG. Las revistas médicas científicas del Perú 1827 - 1996. Lima: Medicina al Día; 1998.

Correspondencia: Charles Huamaní Saldaña

Dirección: Av. Arriba Perú 1154, Villa el Salvador. Lima 42, Perú

Teléfono: (511) 9928-14710 / 287-1397

Correo electrónico: huamani_ca@hotmail.com

La carta fue enviada al autor, quien respondió lo siguiente

\section{RÉPLICA}

\section{[REPLY]}

Oscar G. Pamo-Reyna ${ }^{1}$

Sr. Editor. Respondiendo a la carta del Dr. Charles Huamaní debo manifestar lo siguiente:

1. La selección de las revistas médicas nacionales no fue "por conveniencia" sino que especifiqué claramente las bases de datos que empleé y las revistas mencionadas son las que están circulando con cierta regularidad actualmente ${ }^{(1,2)}$. Como también lo mencioné, hay muchos trabajos nacionales sobre plantas medicinales que se publican en revistas extranjeras y ese es tema para otro estudio. Me circunscribí a las revistas nacionales porque se habla mucho de nuestra milenaria tradición en el uso de plantas medicinales, se expende actualmente numerosas hierbas, raíces y extractos con supuestas propiedades curativas y la realidad es que, tal como lo muestro en mi trabajo, no hay muchos trabajos publicados sobre el tema en nuestras revistas médicas. Más aun, no hay verdaderos estudios definitivos, ya que no hay líneas de trabajo sobre tales propiedades curativas. Tanto así, que ninguna facultad de medicina tiene patentado una planta o derivado con propiedades medicinales.

2. El Dr. Huamaní, gran conocedor en lo referente a las publicaciones científicas médicas nacionales, está muy preocupado porque no incluí a la Revista CIMEL que es de estudiantes de medicina, y a la cual él perteneció. Bien, cuando uno realiza un estudio

\footnotetext{
Profesor Principal, Facultad de Medicina Alberto Hurtado, Universidad Peruana Cayetano Heredia. Médico Internista del Hospital Nacional Arzobispo Loayza. Lima, Perú.
} 